\title{
2 TENDENCIES AND GENERAL REQUIREMENTS FOR VIRTUAL ENTERPRISES
}

\author{
L. M. Camarinha-Matos ${ }^{1 \bullet}, \mathrm{H}$. Afsarmanesh ${ }^{2}$ \\ ${ }^{1}$ New University of Lisbon, Portugal \\ 'University of Amsterdam, The Netherlands
}

The concept of Virtual Enterprise is promoted by the current market and economic conditions worldwide, and facilitated by the recent developments in both information and communication technologies. A large number of international research and development projects are also contributing to the development of basic models and supporting infrastructures. However, there is still a need to overcome many technical, socio-organizational, economic and legal barriers before virtual enterprises can effectively perform during their life cycle. A list of general functional requirements for the various phases of the virtual enterprise's life cycle is presented, based on the identified needs and tendencies in manufacturing systems organization.

\section{INTRODUCTION}

The new market trends, shaped by the globalization of the economy and the formation of large economic blocks, such as the European Union, NAFTA or MERCOSUR, are forcing companies to achieve world class manufacturing levels. Furthermore, requirements for highly flexible / reactive systems, with high quality standards, but constrained by environment friendly requirements, are leading companies to new forms of organizations, relying on privileged cooperation links (Doumeingts et al., 1995).

Nowadays, most of the manufacturing process is not carried on by a single enterprise anymore. Companies feel the need to focus on their core competencies and join efforts with others, in order to fulfill the requirements of the new products / services demanded by the market. In a cooperative networked organization, every

\footnotetext{
- Corresponding author address: Universidade Nova de Lisboa, Quinta da Torre, 2825 Monte Caparica, Portugal, tel. +351-1-2948517, fax +351-1-2941253, e-mail: cam@uninova.pt.
} 
enterprise is just a node that adds some value to the process - a step in the manufacturing / supply chain. Although most classic examples of networked organizations can be found in some particular business domains such as the automotive industry, this tendency is spreading to many other areas including the food and agribusiness industry (Walton, 1996), (Camarinha-Matos, 1997b). The concept of Virtual Enterprise (VE) therefore emerges. A VE materializes by selecting skills and assets from different firms and synthesizing them temporarily into a single functional business entity to respond to business opportunities.

The cooperation among the enterprises involved in a VE is supported by computer networks and other information technology tools. In fact, cooperation between enterprises is not a recent phenomenon at all. For instance, in the civil construction sector there is a long-time tradition of cooperation in consortia to jointly develop large projects. The novelty factor introduced by the virtual enterprise paradigm is the strong use of computer networks and information technologies to support the cooperation among companies. As a consequence of the new facilities offered by these technologies that support both a more effective integration and coordination of activities and the geographical distribution of the involved industries, new forms of organizing the business processes and new ways of working are naturally emerging.

\section{TENDENCIES IN MANUFACTURING SYSTEMS ORGANIZATION}

Several new industrial paradigms have emerged in recent years as an answer to the new challenges of globalization, such as virtual manufacturing, lean enterprise, agile manufacturing, and holonic systems. The introduction of these concepts in the enterprises has made them face successive "waves of restructuring" during the last decades. The emergence of the virtual enterprise paradigm comes in the natural sequence of these restructuring processes enabled by the "explosive" developments in the information and communication technologies. The need to remain competitive in the open market forces companies to seek "world class" status and, therefore, to concentrate on their core competencies while looking for alliances when additional skills / resources are needed to fulfil business opportunities.

Some authors see the roots of this paradigm in early works of economists like Oliver Williamson in the 1970s. Along his very prolific work, and in particular in the "Markets and Hierarchies" (Williamson, 1975) Williamson established the study of Transaction Cost Economics as one of the first and most influential attempts to develop an economic theory of organizations. He defends that manufacturing firms should make much greater use of externally purchased goods and services, rather than internally supplied ones. Williamson discusses the business transaction costs at the same level as the production costs. While production costs are considered as being analogous to the costs of building and running an "ideal" machine, the transaction costs are those that incur by deviation from perfection. For instance he argues that the lack of information about the alternative suppliers might lead to 
paying too high a price for a good or service. Through identifying the important variables that determine the transaction costs, the work of Williamson contributed to the better understanding of business interactions among enterprises.

These ideas had a more evident impact with the booming of the "outsourcing" wave in the 1980s. Outsourcing became very attractive when managers had to reduce the organization overheads and eliminate the internal inefficient services, the so called lean manufacturing, as it transfers the problem to the outside, namely the efficient service providers. For many enterprises, outsourcing some services allows them to concentrate on their core competencies. For others, outside contractors simply provide complementary services for which the company lacks adequate internal resources or skills.

Among many factors that justify the outsourcing strategy, the reduction of costs, and elimination of poor performance units, can be pointed out, particularly in the case of those units that do not represent core capabilities or when better and cheaper alternatives can be identified in the market.

In parallel with the outsourcing tendency, another transformation can be observed in the large companies, that reorganize themselves in terms of their product lines, leading to some "federation" of relatively autonomous departments.

These transformations, putting the emphasis on networking and partnership / cooperation have raised a large interest for new disciplines such as the coordination theory, organizational theory, or sociology of the industrial organizations.

The new forms of cooperation between organizations also raise the need to define new "contract" forms as a way to formalize the specificities of alliances. Based on the classical concept of contract, where the object of agreement and the remedies were clearly and narrowly defined, new forms of contracts are being proposed. For instance, to face the situations of uncertainty where it is impossible to anticipate all contingencies, a form of incomplete contract (neoclassical contract) is developed. These contracts are aimed at regulating long-term relationships, and include the definition of mechanisms for amending the agreements when the (market) conditions change. For other complex and long-term relationships, more than a "legal" contract, it is necessary to have a commitment to cooperate and share risks, the so-called relational contract (Graff, 1995). In the context of distributed business process management, supervision clauses can be used to specify the VE participants' rights and duties in terms of information access for monitoring purposes (Rabelo et al., 1999). However, to derive the supervision clauses based on the rules of the contracts, in order to be handled by a distributed business process management system, is still an open research topic.

Setting the list of requirements for an infrastructure, to support a continuously evolving organization, is quite a challenging task. One approach is to observe tendencies and to study the innovative pilot experiences and infer from them a general list of requirements. A complementary approach is to design plausible futuristic scenarios, and then try to identify necessary functionalities. The MIT Sloan School of Management did a very interesting work on the scenario planning in 
cooperation with more than 300 leading representatives from industry (Laubachet and Malone, 1997). In this study the authors considered two extreme scenarios for the $21^{\text {st }}$ century organizations:

1. Small companies, large networks. Under this scenario, nearly every task is performed by autonomous and small teams, set up as independent contractors or small enterprises, linked by networks, coming together in temporary combinations for various projects, and dissolving once the work is done.

2. Virtual countries. Under this scenario the industry structure is mostly oligopolistic, with a small number of major competitors holding dominant positions, and high entry barriers preventing upstarts from challenging the hegemony of market leaders. These conglomerates would be the result of the merging wave that can be observed in various sectors "in opposition" to the downsizing / outsourcing movement. These large vertically- and horizontally-integrated firms are as powerful and influential as nations.

One important result of this work is a comparative analysis of the business processes for the two scenarios in terms of product development, supply chain management, manufacturing, marketing, finance, and coordination.

These scenarios can be taken as a "delimiting-envelop" as the reality will probably include a mix of organizations.

\section{TECHNOLOGICAL ENABLERS}

\section{Components technologies}

The design and implementation of a supporting infrastructure for VEs can be based on a number of component technologies and paradigms. In our opinion, it is necessary to integrate several existing paradigms and technologies developed in different, but close, disciplines, even more than the effort to define totally new paradigms (Camarinha-Matos and Afsarmanesh, 1998a). In fact, one main reason for the strong increase of activities in VE is the large developments of computer networks related technologies. Figure 1 illustrates the main base components. A synthesis work over these separate components will lead to an effective support environment for VEs.

Furthermore, in order to provide a strong supporting platform, the synthesis process must also include:

-Interoperability and integration of standards, such as STEP (technical product data), EDI (business related transactions), TCP/IP, KQML protocols, etc.

-Support of safety and authentication mechanisms, such as those being developed in the area of Electronic Commerce - cryptography based on symmetric and public keys, digital signature, definition of security rights, etc.

It is important to realize that the various nodes within the VE network wish to, as much as possible preserve their autonomy; in some cases enterprises may even have antagonic goals. Furthermore, as networks are subject to both piracy and temporary 
failures, a VE environment will not be considered a friendly and safe environment. These characteristics must be reflected in the VE supporting platform, through the configuration of its organizational structures, levels of information access rights and information redundancy, and the security protection mechanisms.

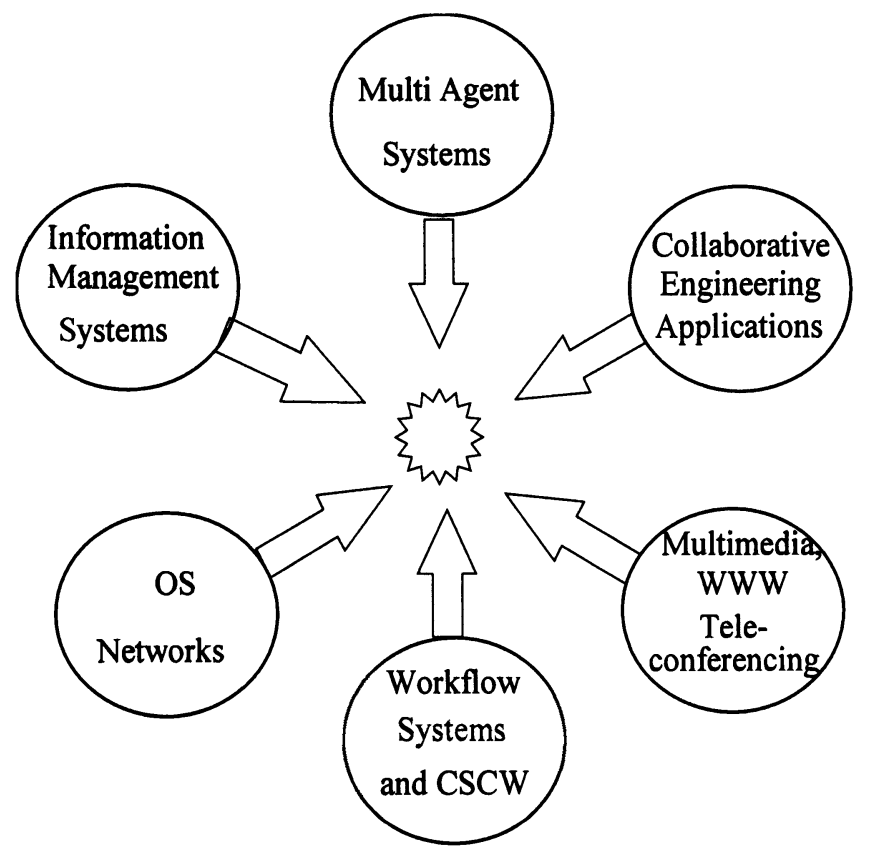

Figure 1 - Some component technologies contributing to a VE environment

Some of the required functionalities in a VE environment involve direct interaction with the human operators, possibly located in different (remote) nodes of the network. HTML-based interfaces with JAVA applets represent a good example implementation approach easily supported by the current browsing technology on the Internet. That is the case, for instance, to support accesses to Electronic Catalogs or supporting the human-launched orders.

In such a case the client node does not need to buy "any extra software" to access its supplier. It just downloads the HTML/Java document from the remote supplier. Nevertheless, due to the need for security and authorized data access mechanisms, it will be necessary to deliver small modules of software, a "cooperation layer", to the client nodes, depending on the implementation strategy chosen for the VE platform.

In many cases the information sources to answer the remote access queries are contained in the legacy systems, e.g. the internal information systems and internal enterprise management software. In principle, a wide range of access to the stored information may be required by enterprises, to be supported for different kinds of environments. 
At one extreme end of the spectrum, it is enough if the VE supporting platform provides the mechanism for homogeneous access to certain specific (pre-defined) kind of information here called "determined-in-advance", from heterogeneous information sources. The mechanism to support such access, is the minimum facility to support information interoperability among enterprises in the VE network. In this case, typically predefined interfaces are developed by each enterprise (e.g. a few web-pages) that can be accessed by other authorized enterprises. At the other end of the spectrum, the opposite case may be required by enterprises within the VE network. This is the case where every enterprise is fully autonomous, and decides to share different parts of its local information with different other enterprises. The decision on what to share and with whom, is decided case by case, and depending on the bilateral interrelationship and contracts of the enterprise with the others. In this case, no predefined set of information can be determined in advance, rather the access to information in other heterogeneous enterprises follows the "determinedon-demand" approach. Such environments require to establish a network of federated databases, where the VE supporting platform needs to provide the mechanism for the definition of information visibility levels and individual information access rights for every other enterprise within the VE network.

For the case of access to information determined-in-advance, the homogeneous access to these heterogeneous information sources can be provided with the webbased technologies. Such a homogeneous access to different sources in a way imitates a form of information integration among these sources. The homogeneous access can be based on a mediator / wrapper approach, where information sources are "wrapped" such that their interfaces to the outside appear uniform, and the mediators are built to mediate the execution (local workflow) of the local tasks in the source nodes. Middleware technology, distributed object framework (such as CORBA, JAVA/RMI, Microsoft/DCOM, or CGI-based extensions to current DBMSs) can be used to support this integration process.

For the case of access to information with determined-on-demand approach, the federated database technology must be applied to support the processing of queries between different heterogeneous enterprises. At the same time, the federatedinformation-integration can support an enterprise with issuing queries at the level of $\mathrm{VE}$, which may in turn involve access to several information sources at different enterprises. The federated-query-processing decomposes such query into subqueries, sent to relevant other enterprises, and merges the results collected from them; and thus hiding from the originating enterprise, the complexity and need to access the distributed information within the VE. As such, federated database systems can properly support the information integration, information sharing, and visibility rights for enterprises in the VE, while preserving their autonomy and without the need for data redundancy for this purpose. It must be noticed however, that federated databases are still under prototypical development within the research and development projects, and are not yet commercial systems.

Some other functions, such as forecasting or product flow tracking, need to have 
access to the information maintained in several different nodes of the VE / supply chain. Clearly, the federated database architecture can properly support any simple or complex information exchange among the VE members. Also the basic general uniform approach described above based on a common middleware technology can be adopted to support these tools, but it is also necessary to install, in each information supplier node, a cooperation layer to interact with the requests issued by remote client tools (Figure 2). Agent-based or distributed object request brokers paradigms can be considered as candidates for this implementation.

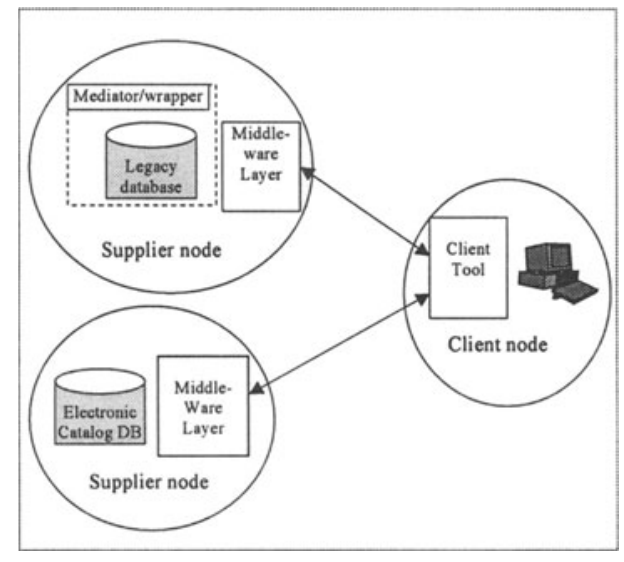

Figure 2 - Homogeneous access to information sources

It is important to notice that the technologies in these areas are still not stable and a lot of advances are expected in the next years, in specific to what relates to the middleware and the safe access mechanism components.

In terms of information sources, it is also important to consider the integration of legacy systems such as PPC / MRP, CAD, information repositories, and other tools that companies already employ. Although these systems are quite heterogeneous, they need to be preserved in the enterprise.

In terms of communication infrastructures, the main aspects to be discussed are the adoption of Internet or VAN (Valued Added Networks), safety and authentication, and the need to face the challenges provoked by the mobile communications, namely the mobile agents in the cooperative environment.

\section{INHIBITORS}

Although several factors such as the market forces and the progress on component technologies represent driving elements, there also exist inhibiting factors or obstacles for the establishment of the VE paradigm. Clearly, these obstacles or 
difficulties can be of a technological nature, but also from a cultural, sociological, or even legal nature.

A main problem is the fact that there is not yet a common reference architecture, widely accepted, to describe VEs. In spite of the many R\&D efforts in different parts of the world, there is a need to reach a larger consensus, as the VEs can be established without geographical barriers. Therefore, geographically-restricted solutions are not good enough. Furthermore, copying the solutions proposed by some advanced societies is not the answer, as it is necessary to take into account the different realities in terms of technological infrastructure, culture, organization, business practices, industry focus, sizes of enterprises, etc.

Understanding distributed business processes and other tendencies in new business practices, or the contractual forms, is a process still in its infancy. The legal framework for the establishment and operation of these networked organizations is not defined yet. Such a framework cannot be limited to a single country when VEs involve members from different countries and even different continents.

Members get involved in such organization on a volunteer basis and directed by some common goals. However, although a VE can be considered as a cooperative system, the individual members are not necessarily by nature cooperative. Individual companies have their own individual goals, sometimes even competing goals, and may naturally show different levels of distrust in openly cooperating with other VE members. At the same time, it is also important to consider that a VE is not an isolated system. Interactions with outside enterprises, via a general-purpose communications infrastructure, will naturally happen, and special care is necessary regarding the non-cooperative (even malicious) actions of some outsiders.

In spite of the recent advances in safe communication mechanisms, especially motivated by the Electronic Commerce area, safety is still an important inhibitor for companies. Knowing that there will be a limit for the level of practical safety, above which the costs are unbearable, it is necessary to create a new culture of risk and to define new mechanisms to cope with that risk, such as new insurance policies.

Cooperation between companies requires some level of mutual trust. Trust building processes, which are gradual and depending on successful past experiences, need to be better understood and properly supported by flexible VE supporting infrastructures. For instance, provision of visibility rights for access to information of other enterprises gives confidence to VE members to trust their cooperation within the VE network.

The socio-organizational issues are almost as important as the technological aspects for the successful implantation of virtual enterprises. However, most of the efforts spent on VE paradigms so far are too technocentric. In other words, these efforts almost ignore the impacts that this new paradigm will have on the way business processes are organized in the enterprise, on the new decision making procedures that are necessary in order to give the enterprise the necessary agility, or on the definition of new job functions with the appropriate re-training of workers. The language, methodologies and approaches used by sociologists, engineers, or 
computer scientists are also quite different, which results a major difficulty in creating a multidisciplinary cooperation platform on this issue.

\section{OVERVIEW OF RESEARCH PROJECTS}

A large number of projects, worldwide, are nowadays addressing different aspects of infrastructures for virtual enterprises. Some of the most representative ones are the North-American NIIIP and the European VEGA, X.CITTIC, PLENT, MARVEL OUS and PRODNET II.

The NIIIP (National Industrial Information Infrastructure Protocols) started in 1994 in the USA, and is perhaps the biggest and most significant project in the VE area. In reality it is more a program than a project. NIIIP intends to support the formation of industrial VEs and to provide technologies that allow VE participants to collaborate within a heterogeneous computing environment. In its general scope, NIIIP addresses the complete VE "Life Cycle": identifying market needs; looking for partners; assisting the negotiation process between partners; and supporting VE instantiation, operation and dissolution. The NIIIP consortium bases its developments on core technologies such as: Internet and related communications facilities and services; Object Management Group (OMG) and related object technology; Standard for the Exchange of Product Model Data (STEP) and related information modeling technologies; Workflow Management Coalition Reference Architecture, etc. NIIIP proposes a reference architecture on top of these technologies and integrates and extends existing protocols. Based on this reference architecture, a number of pilot projects were launched to develop prototype implementations. SMART - Solutions for SME Adaptable Replicable Technology (Barry et al., 1998) is one example of such projects. However NIIIP is based on a very "harmonized" view of the business world, and is too much focused on the North-American reality and interests. According to NIIIP's concepts, all enterprises will work cooperatively, sharing all kinds of resources, including the human ones, which seems to be too optimistic and not in compliance with the current reality in most SMEs in other parts of the world. Therefore, although NIIIP is a base reference architecture to be considered before any new development in the VE area, it cannot be simply copied to other realities.

X-CITTIC (Planning and Control System for Semiconductor Virtual Enterprises) is a European ESPRIT project focused on VEs for the microelectronics sector. In this application domain, the manufacturing process associated to a sales order that can be originated at a customer located anywhere in the world, may be accomplished by manufacturing orders allocation through a globally distributed manufacturing network. X-CITTIC expects to raise, to the virtual enterprise level, some of the techniques currently available in a modern shop floor. Examples of such techniques are the event-driven planning, scheduling, dispatch and orders release.

The goals of MARVEL OUS, an end users driven ESPRIT project, are the 
identification and harmonization of generic requirements for use of advanced IT in manufacturing and engineering across the maritime industry, and building up links to other sectors for mutual benefit. The project intended to guarantee consensus on requirements across the whole range of maritime users and to work closely with the technology providers in order to facilitate the formation of VEs. It also tries to ensure that the end-user requirements are feasible and can be translated into product development.

The Virtual Enterprise using Groupware tools and distributed Architecture (VEGA) ESPRIT project aims to establish an information infrastructure to support the technical and business operations of Virtual Enterprises. Groupware tools and distributed architectures are being developed in compliance with product data technology standardization activities (STEP) and the current trends adopted by the forthcoming international industrial groupware specifications, for example, from the Object Management Group. The approaches and developments resulting from a number of other ESPRIT projects were extended and the strategy for application integration by the distribution of a concurrent access to STEP databases was explored. A complementary route involves the design of a CORBA Access to STEP models (COAST) infrastructure to support the distribution of a product data by means of updated object broker technology. VEGA was focused on the civil construction sector.

PLENT aimed to develop a set of software tools to support coordinated planning in networks of autonomous small and medium size manufacturing enterprises. In the context of this ESPRIT project, the most important problem was not the study of a sophisticated decision-making technique but the definition of a planning policy that is based on well defined, completely visible and strictly applicable rules. This is considered necessary in order to remove the historical distrust between enterprises traditionally in competition with each other, so as to reach an adequate degree of confidence in the network organization.

The Esprit PRODNET II project - Production Planning and Management in a Virtual Enterprise, aimed at the design and development of an open platform to support industrial manufacturing virtual enterprises with special focus on the needs of small and medium size enterprises (SMEs). The basic platform includes:

- Exchange of commercial data via EDIFACT messages;

- Exchange of technical product data using STEP;

- Federated / distributed information management system supporting, not only the administrative information about the VE, but also all the information a node (enterprise) decides to make available to the network;

- Coordination module that handles all cooperation-related events (execution of a local activity flow plan);

- Configurator, allowing the definition and parametrization of the VE and the behavior of each particular node;

- Safe communications;

- Orders status monitoring;

- Quality related information exchange; 
- Extended PPC system, adapted to interact with a VE environment and including the management of incompletely and imprecisely specified orders (along their life cycle);

- Distributed Business Process management, as a first level of coordination of business process execution at the VE level, supporting monitoring, decisionmaking and analysis upon a VE;

- Electronic partners search and selection, based on both private and public directories of suppliers.

Although based on the metal-mechanic sector, the PRODNET II infrastructure is applicable to other sectors, due to the flexibility and configurability being two of its main characteristics. For instance, most components of PRODNET II were easily adopted by the INCO-DC SCM+ project, addressing the supply chain management in agribusiness. SCM+ was a cooperative project funded by the European Commission and involving partners from Europe and Latin America (CamarinhaMatos et al., 1997).

Similar initiatives addressing VE challenges are ongoing in other geographical areas of the world. For instance in the framework of the IMS (Intelligent Manufacturing Systems) program, an initiative involving the USA, European Union, Japan, Switzerland, Canada and Australia, various related works can be found. One example is the GLOBEMAN 21, a large project involving 37 partners from various continents that aimed at creating the new processes and technologies for manufacturing in the twenty-first century including VE-related issues. Within this project 14 industrial demonstrators covering several requirements of a VE platform were developed.

Furthermore, it shall be mentioned that a large number of initiatives are also taking place in the area of Electronic Commerce. From these initiatives several results are of major importance for the establishment of a VE environment, such as the safety and authentication mechanisms, business transactions, electronic catalogs, etc.

\section{GENERAL FUNCTIONAL REQUIREMENTS}

In terms of the computational infrastructure for a VE there is a need for an extensive list of tools to support the various phases of its life cycle (Afsarmanesh, CamarinhaMatos, 1997).

\section{Creation/configuration phase of a VE}

The creation/configuration phase of a VE needs tools for partners search, and for decision support to help the negotiation process and all the dynamics associated to the joining / leaving of enterprises. The main steps include:

i. Search for partners. The process of creation of the VE involves the search and 
selection of partners to be members of the consortium. The problem of finding partners can be decomposed in two groups:

- core partners, responsible for critical components / services;

- subsidiary partners, supplying less important components / services.

For the first group, companies usually have their proprietary list of potential suppliers. For subsidiary partners search however, it may be useful to have some IT-based tools that resort to external directories of suppliers, possibly available on the Internet or other specialized networks.

ii. Negotiation / agreements. At this phase, all potential candidates must send their proposals towards the tender, to the caller/contractor. Additionally some complementary interactions between enterprises are necessary in order to reach a final agreement by both sides. The design of an adequate negotiation protocol and decision support tool is necessary.

iii. Contract awarding and management. There are two stages here that need attention, one is the preliminary contracts signed between partners to write the tender, and the second is the real contract of work that is signed after the tender is selected by the investor. Once the enterprises are selected for a VE, they are entitled to become an official member of that VE. This process involves the contract negotiation between that enterprise and the VE coordinator.

iv. Tender formation. After the preliminary contracts are signed, the VE members will cooperate and jointly write a tender. In the tender the role, responsibilities, and the financial conditions for each enterprise need to be agreed and negotiated by the enterprise and the VE coordinator.

From an "administrative" point of view, there is a need for configuration tools to help in the set up of the infrastructure, according to the agreements made during the contractual phase. For instance, it is necessary to configure the infrastructure to accommodate the particular subset of EDIFACT messages agreed between every two nodes of the VE, or the specific information sharing and visibility rights for VE members.

\section{Operation phase of a VE}

We now assume that a VE is established and started its operational phase. Various levels of interactions among the VE members must be supported. Notice that here the PPC (Production Planning and Control) or ERP (Enterprise Resource Planning) systems of an enterprise are augmented with a supporting layer that supports the enterprise's functionalities as a VE member. The main functionalities include:

i. Basic information exchange interactions. The minimal level of services required in a VE supporting platform are the followings:

- Information exchange mechanisms; to support the exchange of shared/public commercial data (e.g. contract-related interactions), technical data (e.g. 
product models and quality information), general information (e.g. market statistics and catalogues of products/services), etc.

- Interoperability between standards; to support the use of available and emerging diverse information modeling standards, for instance, the EDIFACT and STEP. Protocols and message formats that can combine different standards are necessary. For instance, a specific order, which can be represented in EDIFACT, may reference products whose technical specifications are attached as a STEP model. As a member of a VE, an enterprise must be able to receive, interpret, and generate such multi-standard formatted messages.

- Order status and task performance monitoring; mostly to support the coordination of the VE, but also to support the other node-to-node coworking. For instance, a node (a client), may need to know the status of processing of an order at its current stage in another node (a supplier) in order to prevent any difficulties for processing of its own future orders.

- VE reconfiguration. Along the VE operation many changes can occur, such as partners' replacement, alteration in the monitoring specifications, access rights, etc.

ii. Events / exception handling. As a member of a VE, an enterprise needs to act as an event driven system. Namely, it needs to handle asynchronous events and exceptions either generated inside the company or by other nodes in the network.

iii. Advanced coordination. To properly support the functionality of a VE, and independent of the size of the VE, there is a need for a VE coordinator. The main task of the VE coordinator is the monitoring of the job status (distributed business process) and comparing it to the VE plans as described in the contracts. In the case that an enterprise fails to perform its duties, the VE must be reconfigured to replace the failing enterprise with another one. To support this functionality it is also convenient to have a distributed business process planning / modeling tool that allows for re-planning and re-scheduling of business processes.

iv. Material / services related aspects. It is important to support functionalities necessary to represent and monitor the flow of products and services through the VE network. Some required functionalities include:

- Materials/services-flow management. Identification, representation and monitoring of all materials/services flows within the network.

- Logistics. Planning of the transportation, inventory and warehousing. This includes route planning, vehicles / crew assignment, distribution sequencing, etc., for instance all activities which provoke materials/services flow between a point of origin and a point of consumption/destination, including the subsets of supply logistics, production logistics, distribution logistics and waste logistics.

- Forecasting. The use of electronic links to transmit information from points of sale to the production units, and suppliers to clients, combined with the historic 
data, will allow the implementation of material / services related forecasting functions.

- Specific information flows related to product (bar coding, POS). In particular, it is important to understand and coordinate the interactions between materials/services flows and information flows and the multiple bar codes used.

\section{Dissolution of a VE}

This is the least studied phase of the VE life cycle in the current literature, but some tools are certainly required to support the following main aspects among others:

- Definition of general liabilities upon the dissolution of the VE;

- Keeping track of the individual contributions to a product / service that is jointly delivered; namely in terms of the quality and product life cycle maintenance;

- Redefinition / ceasing information access rights after ceasing the cooperation; and

- Assessing the performance of partners, generating information to be used by partners' selection tools in future VE creation.

\section{New emerging services}

It is important to notice that the VE paradigm is not an isolated phenomenon. Many new other services are rapidly being proposed over the Internet and some of them contribute to the functionalities required for virtual enterprises. One important related area is the Electronic Commerce that proposes solutions on important issues such as:

- Organization and publication of electronic catalogues and related mechanisms;

- Security mechanisms, namely to support interchange of payments related information;

- Advanced and customizable search engines, some of them based on mobile software agents; and

- Legal issues related to electronic-based business transactions.

\section{Legacy systems}

Finally it is important to mention that coping with legacy systems is a mandatory requirement for any VE-supporting infrastructure. The strong reliance on standards will contribute to facilitate the interfacing of existing applications with the VE infrastructure, but unfortunately not all classes of information that need to be exchange among VE nodes are covered by existing standards. Initiatives of groups of application developers such as the Workflow Management Coalition (WfMC, 1994) or the Open Applications Group (OAG, 1997) can also contribute to facilitate this process. In general, it is necessary to develop some interface / mapping layer, at each enterprise, to adequately have this enterprise interacting with the VE, via the VE infrastructure.

It shall also be noticed that most legacy applications were designed for a local 
operation (enterprise-centered) and to be operated by humans. In order to have these applications supplying information to or consuming information from the VE network, it is clearly necessary to extend their functionality.

\section{CONCLUSIONS}

The development of the VE paradigm is being strongly pushed by the economic factors worldwide and facilitated by the recent developments in the information and communication technologies. There is however a large number of open issues and obstacles in spite of the growing number of international research and development projects in this area.

This paradigm represents an opportunity and a challenge for industrial enterprises worldwide justifying a more "integrated" and not geographically bounded approach to the development of open infrastructures adaptable to the different socio-economic and technologic realities.

A large number of general functional requirements for a VE supporting infrastructure, covering the various phases of the VE life cycle, can already be identified, some of them being extensively investigated by many projects. Some of these functional requirements are however, still unclear, requiring further investigation and a clear multi-disciplinary approach involving not only the technology-oriented aspects but also the socio-organizational and legal issues.

\section{Acknowledgements}

This work was funded in part by the European Commission, Esprit programme within the PRODNET II project. The authors also thank the valuable contributions from the consortium partners: CSIN (P), ESTEC (P), HERTEN (BR), Lichen Informatique (F), MIRALAGO (P), ProSTEP (D), Uninova (P), University of Amsterdam (NL), Universidade Federal de Santa Catarina (BR), and Universidade Nova de Lisboa (P).

\section{REFERENCES}

1. Afsarmanesh, H. ; Camarinha-Matos, L.M. - Federated Information Management for Cooperative Virtual Organizations, DEXA'97 $-8^{\text {th }}$ Int. Conf. On Database and Expert Systems Applications, Lecture Notes in Computer Science Nr. 1308, Springer-Verlag, 1997.

2. Barry, J.; Aparicio, M.; Durniak, T.; Herman, P.; Karuturi, J.; Woods, C.; Gilman, C.; Ramnath, R. NIIIP-SMART: An investigation of Distributed Object Approaches to support MES development and deployment in a Virtual Enterprise, $2^{\text {nd }}$ IEEE International Enterprise Distributed Computing Workshop (EDOC'98), 2-5 Nov 1998.

3. Camarinha-Matos, L.M.; Afsarmanesh, H. - Cooperative Systems challenges in Virtual Enterprises, Proc. CESA'98 - IMACS/IEEE Multiconference on Computational Engineering in Systems Applications, Vol. 3, pp. 403-408, Nabeul-Hammamet, Tunisia, 1-4 Abr 98. 
4. Camarinha-Matos, L.M.; Afsarmanesh, H. - Virtual Enterprises: Life cycle supporting tools and technologies in Handbook of Life Cycle Engineering: Concepts, Tools and Techniques, A. Molina, J. Sanchez, A. Kusiak (Eds.), Chapman and Hall, June 1998, ISBN 0-412-81250-9.

5. Camarinha-Matos, L.M.; Carelli, R.; Pellicer, J.; Martin, M. - Towards the virtual enterprise in food industry, Proc. ISIP'97, OE/IFIP/IEEE Int. Conf. on Integrated and Sustainable Industrial Production, Chapman \& Hall (Re-Engineering for Sustainable Industrial Production), Lisbon, Portugal, May 1997, ISBN 0-412-79950-2, pp. 73-84.

6. Doumeingts, G.; Ducq, Y.; Clave, F.; Malhene, N. - From CIM to Global Manufacturing, Proceedings CAPE'95 - Computer Applications in Production Engineering, Chapman \& Hall, 1995.

7. Graff, J. - An introduction to the work of Oliver Eaton Williamson, June 1995, www.iems.nwu.edu/ iamison/quartz/will-wp.html.

8. Laubacher, R. J.; Malone, T. W. - Two Scenarios for the $21^{\text {st }}$ Century Organization: Shifting networks of small firms or All-encompassing "Virtual Countries", MIT Sloan School of Management, Working paper 21C WP \#001, Jan 1997.

9. NIIIP - The NIIIP Reference Architecture, 1996, http://www. niiip.org.

10. OAG - Open Applications Integration White Paper, Open applications Group, 1997.

11. Rabelo, R. J.; Klen, A. P.; Spinosa, L. M.; Ferreira, A. C. - Agile Supply-Chain Coordination in the Virtual Enterprise Environment, Proceedings of the $4^{\text {th }}$ IFAC/IFSA Brazilian Symposium on Intelligent Automation - SBAI'99, São Paulo, Brazil, September 1999.

12. Walton, J.; Whicker, L. (1996) - Virtual Enterprise: Myth \& Reality, J. Control, Oct. 96.

13. Williamson, O. E. - Markets and hierarchies: analysis and antitrust implications: A study in the economics of internal organization, Free Press, 1975.

14. WfMC - Workflow Management Coalition, The Workflow Reference Model - Document Number TC00 - 1003, Issue 1.1, Brussels, Nov 94. 